\title{
Effects of drought stress on photosynthesis and chlorophyll fluorescence images of soybean (Glycine max) seedlings
}

\author{
Wensen Wang ${ }^{1,2,3}$, Cheng Wang ${ }^{2,3,4}$, Dayu Pan ${ }^{2,3,4}$, Yakun Zhang ${ }^{2,3,4}$, Bin Luo ${ }^{2,3,4 *}$, Jianwei $\mathrm{Ji}^{\mathrm{i}^{*}}$ \\ (1. College of Information and Electrical Engineering, Shenyang Agricultural University, Shenyang 110866, China; \\ 2. Beijing Research Center of Intelligent Equipment for Agriculture, Beijing 100097, China; \\ 3. National Research Center of Intelligent Equipment for Agriculture, Beijing 100097, China; \\ 4. Beijing Key Laboratory of Intelligent Equipment Technology for Agriculture, Beijing 100097, China)
}

\begin{abstract}
The main purpose of this research is to provide a theoretical foundation for the screening of drought-resistant soybean varieties and to establish an efficient method to detect the PSII actual photochemical quantum yields efficiently. Three soybean varieties were compared in this experiment after $15 \mathrm{~d}$ when they were planted in a greenhouse. These varieties were then exposed to light drought stress (LD) and serious drought stress (SD) conditions. With five times' measurement, chlorophyll fluorescence and soil-plant analysis development considered as the main basis for this study. Several parameters in SD conditions significantly reduced, such as net photosynthetic rates $(P n)$, stomatal conductance (Gs), PSII primary light energy conversion efficiency $(F v / F M)$, PSII actual photochemical quantum yields $[Y(I I)]$, photochemical quenching coefficient $(q P)$ and non-photochemical quenching coefficient $(q N)$. The soybeans in the seedling stage adapted to the inhibitory effect of drought stress on photosynthesis through stomatal limitation. Under serious drought stress, non-stomatal limitation damaged the plant photosynthetic system. The amplitudes of $P n$ and $Y(I I)$ of drought-resistant Qihuang 35 were lower than those of the two other varieties. Based on the data of this study, a new method had been developed to detect $Y$ (II) which reflected the photosynthetic capacity of plant, $R=0.85989, u=0.048803$ when using multiple linear regression, and $R=0.84285, u=0.054739$ when using partial least square regression.
\end{abstract}

Keywords: soybean seedling, drought stress, photosynthetic parameters, chlorophyll fluorescence parameters, chlorophyll fluorescence images

DOI: $10.25165 / \mathrm{j} . \mathrm{ijabe} .20181102 .3390$

Citation: Wang W S, Wang C, Pan D Y, Zhang Y K, Luo B, Ji J W. Effects of drought stress on photosynthesis and chlorophyll fluorescence images of soybean (Glycine max) seedlings. Int J Agric \& Biol Eng, 2018; 11(2): $196-201$.

\section{Introduction}

Drought, a severe natural disaster, is characterised by insufficient rainfall capacity to support plant growth and yield formation and this phenomenon eventually causes plant damage and even death. Drought exacerbates because of economic development, population growth and environmental degradation ${ }^{[1-3]}$. For as much as populations will exceed 9 billion in $2050^{[4]}$, water consumption in industrial, agricultural and other fields will increase. Therefore, water resource scarcity will become severe, or in other words, drought stress will seriously threaten the growth and reproduction of crops, even survival and development of human. The damage caused by drought to plants is primarily attributed to the inhibition and disruption of photosynthesis, which is the main mechanism of plant growth and maintenance of natural

Received date: 2017-03-31 Accepted date: 2018-02-25

Biographies: Wensen Wang, $\mathrm{PhD}$ candidate, research interests: agricultural biological sensors, Email: 278978916@qq.com; Cheng Wang, PhD, Professor, research interests: agricultural intelligent equipment, Email: wangc@ nercita.org.cn; Dayu Pan, Master, research interests: sensor technology, Email: pandy@nercita.org.cn; Yakun Zhang, PhD candidate, research interests: agricultural biological sensors, Email: zhangyakun2011@163.com.

*Corresponding author: Bin Luo, PhD, research interests: agricultural information technology. Room 606, Building B, Beijing Nongke Mansion, No.11 Shuguang Huayuan Middle Road, Haidian District, Beijing 100097, China. Tel: +86-13488795634, Email: luob@nercita.org.cn; Jianwei Ji, PhD, Professor, research interests: agricultural biological sensors. Room 321, Building of College of Information and Electrical Engineering, Dongling Road No.120. Shenyang 110866, China. Tel: +86-13019346595, Email: jijianwei@126.com. environments, and it threats to the growth and yields of hydrophilic plants $^{[5]}$, including soybean. Originating from China, soybean (Glycine max) is an essential grain and oil crop rich in protein and unsaturated fatty acids without cholesterol. Also, soybean is regarded as a vital industrial raw material and economic crop worldwide, as this crop has developed rapidly on a global scale and soybean yields in America and Brazil have exceeded those in China since the mid-20th century ${ }^{[6]}$. Thus, recognition and development of preventive measures against this condition, and screening of drought-resistant varieties are the primary steps to ensure soybean yield $^{[7]}$. Accordingly, identifying suitable seed selection method for cultivation or optimization of soybean varieties and developing detecting or stress-alert methods can improve economic benefits, optimise water resource distribution, and even relieve environmental degradation.

Through plant leaf is the main functional organ of photosynthesis, previous studies had mainly analysis the photosynthesis of leaves by plant leaf gas-exchange ${ }^{[8,9]}$. Zivcak et al. ${ }^{[10]}$ revealed that drought stress damages not only photosynthesis-related components, but also photosynthetic organs; Singh and Raja ${ }^{[11]}$ demonstrated that plants elicit different responses and employ various feedback mechanisms in response to different degrees of drought stress. As a non-destructive evaluation method, chlorophyll fluorescence can accurately and rapidly measure plant health status, provide information on photosynthetic electron transport under drought stress, and examine stress factors, including drought and diseases ${ }^{[12-14]}$. Although chlorophyll fluorescence images contain larger amounts of 
information than parameters and are more adaptable to modern unmanned high throughput measurement ${ }^{[15]}$, the number of studies focused on chlorophyll fluorescence images is less than expect. Moreover, despite influence of drought stress on soybeans in the seedling stage has been described with different parameters, the effects of drought stress on chlorophyll fluorescence and its image combined with photosynthetic parameters of soybean leaf have not yet to be comprehensively analysed. Therefore, the main objective of this study was to investigate the relations between drought stress and the plant photosynthetic physiological ecology, besides develop new technologies to improve the efficiency and lower the artificial error of plant detection, with mainly researching the effects of drought stress on chlorophyll fluorescence and photosynthesis with figures and images.

\section{Materials and methods}

\subsection{Experimental materials and processing}

Located at Xiaotangshan National Precision Agricultural Research Demonstration Base $\left(40^{\circ} 18^{\prime} \mathrm{N}, 116^{\circ} 45^{\prime} \mathrm{E}\right)$, this experiment was implemented in a greenhouse which was proper ventilation for $24 \mathrm{~h}$ and had daytime temperature ranging from $23^{\circ} \mathrm{C}$ to $35^{\circ} \mathrm{C}$. The experimental soil type was a mixture of ordinary sandy soil and matrix at a proportion of 1:1. Its nutrient contents were organic matter content $(8.76 \%)$, total nitrogen content $(0.31 \%)$, available potassium content $(110 \mathrm{mg} / \mathrm{kg})$, available phosphorus content $(34.1 \mathrm{mg} / \mathrm{kg})$ and the $\mathrm{pH}$ of it is 7.4 . After aerating, sterilizing, sieving through an $8 \mathrm{~mm}$ screen and placing into bottom-punched holes pots (specifications was $22.25 \mathrm{~cm} \times 20.00$ $\mathrm{cm}$ (calibre $\times$ depth), weight was $0.24 \mathrm{~kg}$ ) the soil, each pot was filled with $4.85 \mathrm{~kg}$ soil and the maximum water content of it was $41.60 \%$. Experimental soybean varieties included three varieties widely cultivated in China, 'Zhonghuang 13' (No. Soybean 2001008), 'Qihuang 35' (No. S2015005) and 'Hedou 12' (No. S2002012).

In this experiment, soybean seeds were sown on August 16, 2016. Five holes were in each pot and two seeds were in each hole with a seedling depth of $2.5 \mathrm{~cm}$. After, the growth patterns in all of the pots were consistent $15 \mathrm{~d}$ after sowing at August 31 . Three healthy soybean seedlings were cultured and subjected to water treatment which moisture supplementation was implemented at 17:00 each day. Furthermore, a weighing method was to maintain the soil moisture content at the designed gradient which included control (CK, sufficient water supply), with soil relative water content of $75 \%-65 \%$; light drought stress (LD), with soil relative water content of $45 \%-55 \%$; and serious drought stress (SD), with soil relative water content of $25 \%-35 \%$. Just before induced stress, the first group of data had been measured. The following data was obtained once every $5 \mathrm{~d}$, respectively at August 31, September 4, September 9, September 14 and September 19. In total, the stress continued $20 \mathrm{~d}$, and five groups of data were established. Then, rehydration treatment was implemented after stress, and the number of podding was determined on October 10 , 2016.

\subsection{Measuring items and methods}

\subsubsection{Measurement of gas exchange parameters}

Li-6400 portable photosynthesis meter (LICOR Inc., USA) was adopted to conduct measurement of gas exchange parameters, with the measuring time between 8:30-10:00. Measuring position was placed in the centre that deviates for $1 \mathrm{~cm}$ on the $3^{\text {rd }}$ layer of leaf layer, and the measuring area was $2 \mathrm{~cm} \times 3 \mathrm{~cm}$. After the indices were stabilised, the measured values were recorded five times every $2 \mathrm{~s}$. The instrument used was an open-type gas circuit, while the light source used was natural light in the greenhouse, and the light intensity was about $300 \mu \mathrm{mol} /\left(\mathrm{m}^{2} \cdot \mathrm{s}\right)$. Following parameters were measured: leaf net photosynthesis rate $(P n)$, stomatal conductance $(G s)$, intercellular carbon dioxide concentration $(\mathrm{Ci})$ and environmental carbon dioxide concentration $(\mathrm{Ca})$. In addition, stomatal threshold value was calculated according to the following formula: $L S=1-\mathrm{Ci} / \mathrm{Ca}$.

2.2.2 Measurement and acquisition of chlorophyll fluorescence parameters and images

The vision sensor (pixel size was $640 \times 480$ ) of this study is provided by Zhejiang University, whose technical specifications are similar to Multi-color FluorCam. The mechanism design and the electrical system design is own research and development. With the measuring time set between 20:30-0:30 (the dark adaptation time was longer than $1 \mathrm{~h}$ ) and the measuring position placed at the centre and deviating for $1 \mathrm{~cm}$ on the $2^{\text {nd }}$ layer or $3^{\text {rd }}$ layer of soybean leaf. Measurements were repeated for five times. To evaluate dark adaptation, light with pulse width being $40 \mu \mathrm{mol} /\left(\mathrm{m}^{2} \cdot \mathrm{s}\right)$ was applied for measuring and minimum fluorescence under dark adaptation $(F o)$ values were recorded. Photochemical light $\left(400 \mu \mathrm{mol} /\left(\mathrm{m}^{2} \cdot \mathrm{s}\right)\right)$ with light intensity was turned on with saturated flashing $\left(800 \mu \mathrm{mol} /\left(\mathrm{m}^{2} \cdot \mathrm{s}\right)\right)$ applied every 5 s. After 15 complete pulse measurements, maximum fluorescence (with an average value of the last three times was taken) $\left(F m^{\prime}\right)$ and steady-state fluorescence $(F s)$ were recorded. Photochemical light was turned off, with measurements for minimum fluorescence $\left(F O^{\prime}\right)$ under light adaptation recorded. Fluorescence parameters were calculated according to the following equations:

PSII primary light energy conversion efficiency:

$$
F v / F m=(F m-F o) / F m
$$

PSII actual photochemical quantum yield:

$$
Y(I I)=\Phi P S I I=\left(F m^{\prime}-F s\right) / F m^{\prime}
$$

Photochemical quenching coefficient:

$$
q P=1-\left(F s^{-} F o^{\prime}\right) /\left(F m^{\prime}-F o^{\prime}\right) \text { or } q L=q P \cdot F O^{\prime} / F S
$$

Non-photochemical quenching coefficient:

$$
q N=1-\left(F m^{\prime}-F o^{\prime}\right) /(F m-F o) \text { or } N P Q=F m / F m^{\prime}-1
$$

The images were data of $F v / F m, Y(I I), q P, q N$ and $Y(N O)$ (quantum yield of non-regulatory energy dissipation, $Y(N O)=$ $1 /[N P Q+1+q L(F m / F o-1)]$, respectively).

2.2.3 Modelling of the method using image to predict $Y$ (II)

In this study, the process of modelling has been divided into two stages, image pre-processing and mathematical modelling. Firstly 'Mean Filtering Algo' is used in the denoise stage and using area threshold value to segment images. Afterwards, $R, G, B$, $G R A Y, H, S, V, R / G, G / R, R /(G+B), G /(R+B), B /(R+G), r, g, b$, NID, Exr, Exg information of each image has been extracted as the database. Through the PCA dimensionality reduction of the database, five characteristic values of each graph has been the explanatory variable of this study and five characteristic values occupy more than $98 \%$ information of the image. Ultimately, the actual values had been defined as interpreted variation of predicting $Y(I I)$.

\subsubsection{Data arrangement and analysis}

Several softwares were used to arrange and analyse the data: Microsoft Excel 2007 was used to conduct statistical data analysis. SPSS v22.0 was used to conduct significance analysis $(p<0.05)$. Origin 9.1 was used to make function plots. Microsoft Word 2007 was used to make the table of values. Matlab 2012b was 
used to mathematical modeling.

\section{Results and discussion}

\subsection{Effects of drought stress in the seedling stage of soybean} on gas exchange parameters

Overall, the trend of net photosynthetic rate $(P n)$ of the three varieties under normal water supply treatment (CK) firstly appeared low and but then slowly rose (Figure 1a). After day 10 of SD stress treatment, Pns were significantly lower than CK groups $(p>0.05)$. By comparison, the decrease of Hedou12 was greater than other varieties, but Qihuang35 was the opposite, decreased lower than the other varieties. The overall trend of Gs under CK treatment was different from the variation trend of $P n$ (Figure 1b). Under SD treatment, the influence of drought stress on $G s$ of the three varieties was identical with its influence on $P n$, and their difference was identical. Under drought stress treatment, variation of stomatal threshold value $L S$ of the three varieties was obvious that all three varieties under SD treatment rapidly increased on day 5 and decreased on day 20 while they were significantly higher than those under CK treatment, the general trend is opposite to Gs (Figure 1c).
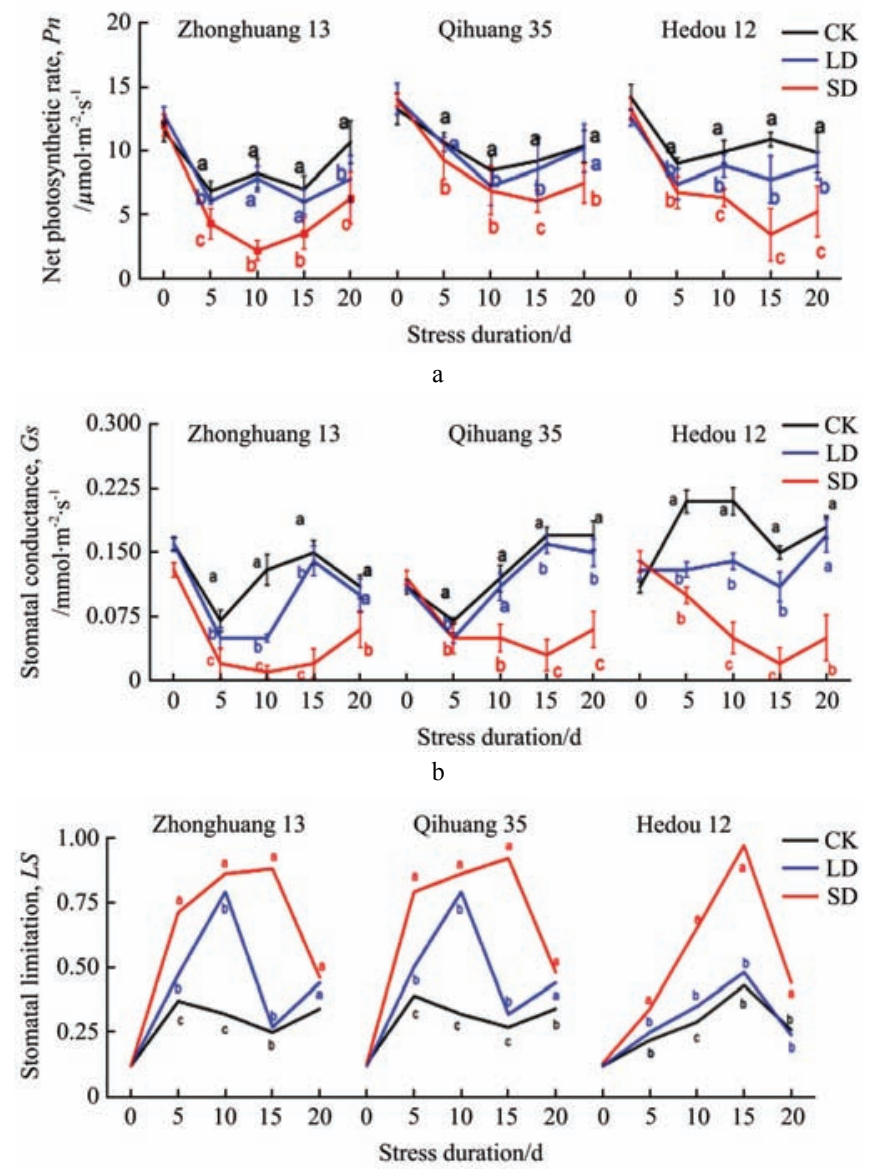

Figure 1 Changes of gas exchange parameters of Zhonghuang 13, Qihuang 35 and Hedou12 after drought stress

Usually, $P n$ decreases because of stomatal limitation or non-stomatal limitation ${ }^{[16]} P n$ decreases by regulating or closing the stoma in stomatal limitation and the viability of photosynthetic cells; organs is damaged in non-stomatal limitation. Testing whether the main cause for the decreased $P n$ is stomatal limitation which is universally applicable to the method proposed by Farquhar and Sharkey ${ }^{[17]}$. Using the trends of Gs and intracellular concentration of $\mathrm{Ci}$, if the two presents positive correlation when $P n$ decreases, then the reason for $P n$ decrease is stomatal limitation if the two showed no correlation or are contrary, then it is non-stomatal limitation. Lu et al. ${ }^{[18]}$ indicated that the reason for the decreased $P n$ in soybeans under serious drought stress is mainly non-stomatal limitation. Conversely, Guo ${ }^{[19]}$ indicated that $P n$ of soybeans under serious drought stress decreases because of stomatal limitation and this conclusion was relatively similar to our findings. In order to avoid the effects of fluctuating environmental concentrations of $\mathrm{Ca}$ on $\mathrm{Ci}$ measurement ${ }^{[20]}, L S$ had been used to replace $C i$ to conduct photosynthesis parameter analysis in this study. Therefore, $P n$ of the three varieties decreased under drought stress treatment because of stomatal limitation after trend analysis. Although $L S$ decreased and its trend was different from that of $G s$ in the terminal stage of stress (15-20 d), the decreasing range of $L S$ was excessively larger than that of $G s$ obviously. These findings indicated that drought stress damaged the photosynthetic organs of plants because of extended stress duration, and plants would lose their ability to regulate their stoma, non-stomatal limitation also limited photosynthesis finally. Through the research of the relationship between stoma and stress and the analysis of this study and other literatures, the way to reduce photosynthesis is not only influenced by stress intensity, but also by other objective factors, such as variety, stress duration and so on ${ }^{[16,18,19]}$.

\subsection{Effects of drought stress on chlorophyll fluorescence} parameters and images of soybean

Drought stress could significantly decrease the primary light energy conversion rate $(F v / F m)$ of experimental samples (Figure 2a). Variation rule of PSII actual photochemical quantum yields $[Y(I I)]$ of the three varieties in the CK group initially decreased but then slowly increased (Figure $2 b$ ) and these were similar to $P n$ variation trends. Moreover, $Y(I I)$ s of three varieties under SD treatment were lower than those in $\mathrm{CK}$ group as drought stress lengthened and trends basically maintained identical. For the three varieties, the overall variation trends of their photochemical quenching coefficient $(q P)$ under $\mathrm{CK}$ treatment firstly increased and then slowly decreased with plant growth (Figure 2c). Under LD treatment, the $q P \mathrm{~s}$ of three varieties almost showed no significance difference than those under $\mathrm{CK}$ treatment and maintained consistent trends. However, for the $q P$ under SD treatment, they were significantly lower than those under CK treatment. Under $\mathrm{CK}$ treatment, the variation of non-photochemical quenching coefficient $(q N)$ was similar to that of $q P$ (Figure $2 \mathrm{~d}$ ).

Light energy absorption and transformation by plants are mainly divided into three closely related parts: chlorophyll fluorescence, $q P$-related photosynthetic electron transport and $q N$-related heat consumption ${ }^{[21,22]}$. In this experiment, drought stress reduced $F v / F m, q P$ and $q N$ which respectively reflects the integrity or health of a function in a plant leaf ${ }^{[23-25]}$, and these are of a relatively weak regularity with the rank of stress. In contrast, $Y(I I)$ is the expression of the results of all functional cooperation ${ }^{[26]}$, and basically consistent with the $P n$ trend. Besides, stress on plant photosynthesis in the study of photosynthetic efficiency can make effective evaluation on plant photosynthesis, although different plant species and varieties have different mechanisms and functions of stress resistance. Therefore, in the study of plant stress tolerance, using $Y(I I)$ to evaluate the plant's overall stress or to stress early warning is superior to other parameters. 


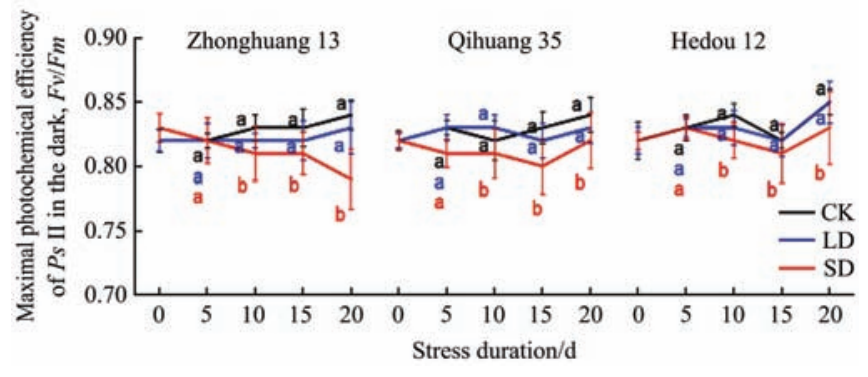

a

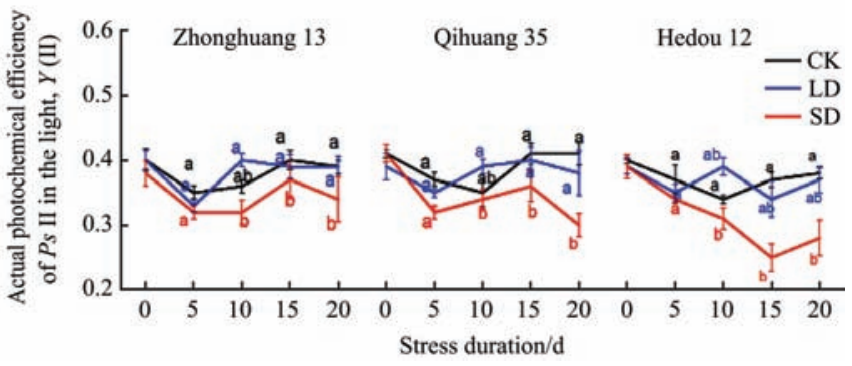

b

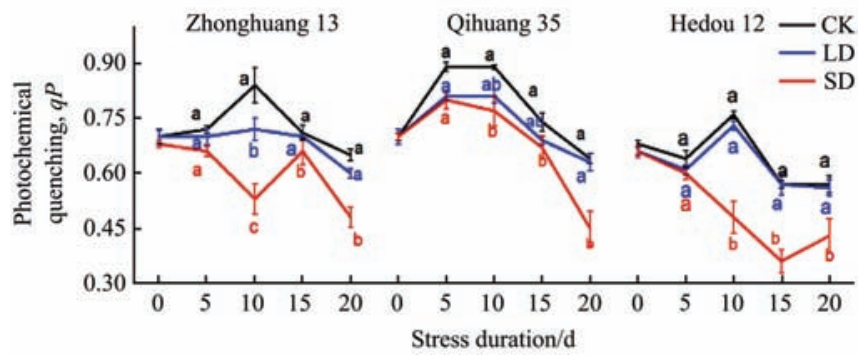

$\mathrm{c}$

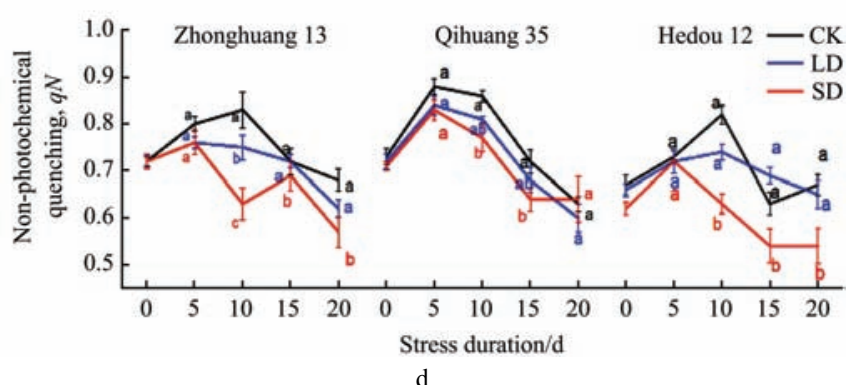

Figure 2 Changes of chlorophyll fluorescence parameters of Zhonghuang 13, Qihuang 35 and Hedou 12 after drought stress

\subsection{Effects of drought stress on the number of podding of} soybean

Under LD treatment, the average podding number of Zhonghuang 13 decreased the lightest, and was 12.77 that reached 95.77\% of it under the CK treatment (13.33). By contrast, under SD treatment, average podding number of Qihuang35 $(=29.59 \%$ $\mathrm{CK}$ ), was showed to higher than under other two varties. Moreover, for Hedou 12, the podding quantities under CK, LD and SD treatments were $11.84(100 \%), 8.16(68.90 \%)$, and 1.74 $(14.65 \%)$ respectively, which whether under LD or SD treatment, the podding number decreased the most among the three varieties (Figure 3).

Drought stress in the seedling stage of soybeans reduced the accumulation rate of dry matter and influenced the podding rate to reduce soybean yield directly. Qihuang 35 under serious drought stress exhibited a healthy status and a superior photosynthetic capacity to the two other varieties, and the decreasing rate of $P n$ and $Y(I I)$ of Qihuang 35 were the lowest. Thus, combined with the production, Qihuang 35 exhibited a certain degree of drought resistance. However, for Zhonghuang 13 under LD treatment, its low $Y(I I)$ range were smallest and podding quantity also decreased at the lowest rate. To sum up, the yield of soybean is in accordance with the performance of $P n$ and $Y(I I)$ when faced drought stress. Therefore, the anti-stress capacities of various drought gradients should be considered and an optimum seed selection scheme should be obtained to select drought-resistant varieties of soybeans, and the $P n$ and $Y(I I)$ can reflect the output of soybeans to a certain extent.

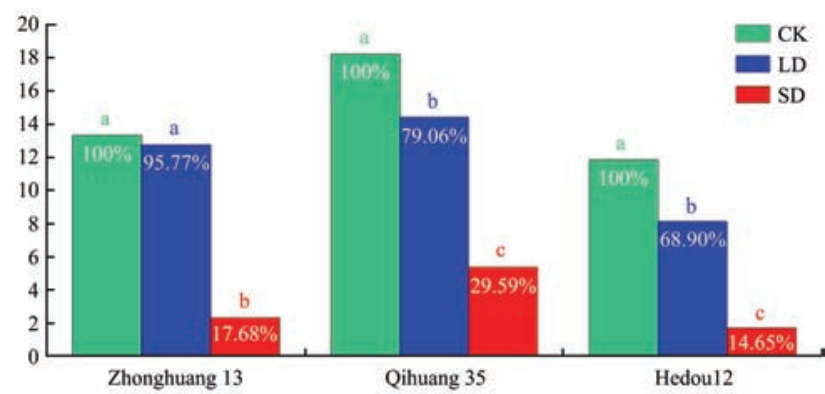

Figure 3 Changes of podding quantities of Zhonghuang 13, Qihuang 35 and Hedou12 after drought stress

\subsection{Acquisition and analysis of chlorophyll fluorescence image}

For a single variety, images of the lightness of $F v / F m, q P, q N$ and $Y(I I)$ decreased after day 20 of drought stress, and these findings were similar to those of chlorophyll fluorescence parameters, but the lightness of quantum yield of non-regulatory energy dissipation[Y(NO)] increased through comparison (Figure 4).

总
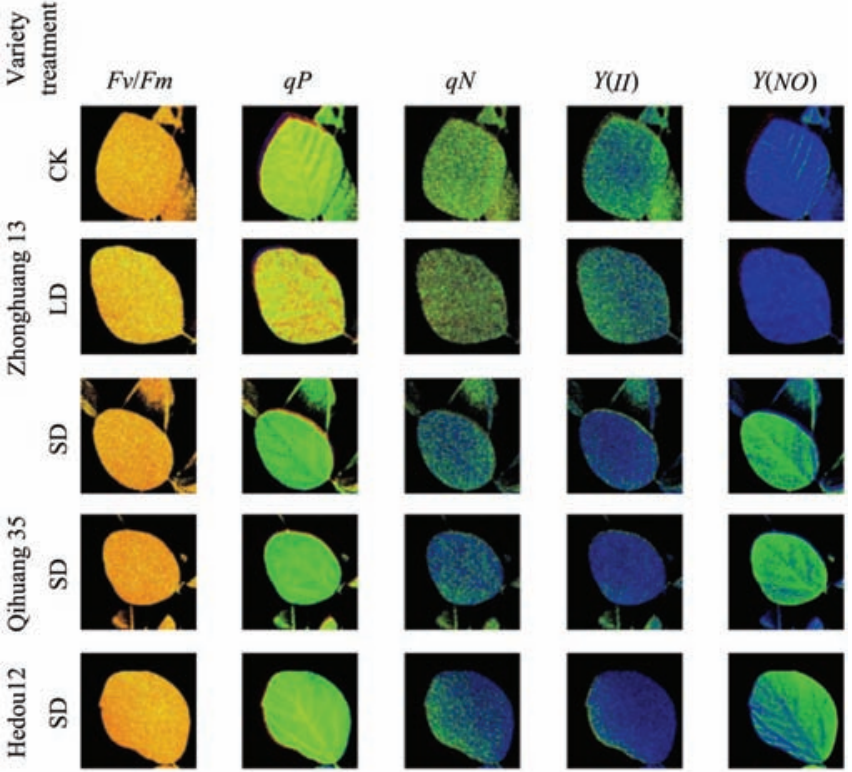

Figure 4 Changes of chlorophyll fluorescence parameter images of Zhonghuang 13, Qihuang 35 and Hedou 12

Through the steps of image preprocessin, most imformation of the images had been saved and most interference information had been removed, including incomplete leaves, stems and noise (Figure 5). Two modeling methods were developped, $R=0.85989$, $u=0.048803$ with multiple linear regression (MLR), and $R=0.84285$, $u=0.054739$ with Partial Least Square Regression (PLSR). After, selected two leaves to test the algorithms, which details can be shown in Table 1. 


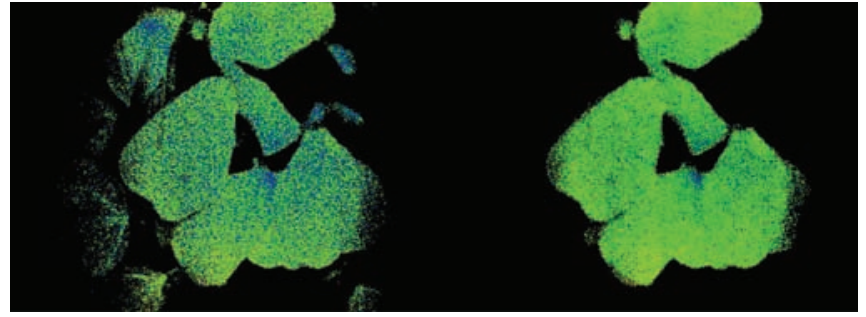

Figure 5 Original chlorophyll fluorescence images and the images after treatment

Table 1 Test of algorithms precided $Y(I I)$

\begin{tabular}{ccccc}
\hline \multirow{2}{*}{ Algorithm } & In the modelling & Actual value & Predicted value & Relative error \\
\hline \multirow{2}{*}{ MLR } & Yes & 0.4032 & 0.4208 & $4.37 \%$ \\
& No & 0.3905 & 0.4122 & $5.56 \%$ \\
\hline \multirow{2}{*}{ PLS } & Yes & 0.4032 & 0.4383 & $8.7 \%$ \\
& No & 0.3905 & 0.3775 & $-3.32 \%$ \\
\hline
\end{tabular}

Manual measurement must be carried out with artificial error in processing measurement of photosynthesis. However, with the development of communication technology and mathematical modelling, the original detection method (point measurement), such as the report based on the analysis of woody plants by Salvatori ${ }^{[27]}$ and the report focused on fruit by Hou et al ${ }^{[28]}$, may not present the information of the whole leaf of plant including image evidence, nor fit the high throughput phenotypes. Besides, the studies using fluorescence graphs mostly focused on fluorescence spectrums ${ }^{[29,30]}$, which can only presented the instantaneous value of fluorescence. In addition, the parameters should be compared with those in the CK group to determine whether plants were influenced by drought stress. Fluorescence images revealed that the variation of $Y(N O)$ under no stress or under light stress was not evident, and by comparison, lightness significantly increased under relatively serious drought stress. This parameter image could be identified even without comparing it with CK group or controlling the time at which soybean plants were under serious drought stress. In addition, relative to chlorophyll fluorescence parameters, images revealed information regarding the whole leaf and these images illustrate the responses of leaves to drought stress ${ }^{[14]}$. Therefore, this study developed a new method that realized acquiring chlorophyll fluorescence information directly from the images, to fit automation technology and to present more plant physiological information, by using precise measurement of chlorophyll fluorescence parameters and chlorophyll fluorescence images for modelling. This study used $Y(I I)$ as an example for modeling that the prediction success rates had been acceptable, and this method can fit the high throughput plant phenotypic platform, unmanned factory, stress alert system and UAV detection. Further research that using more algorithms and using more data is needed to improve the accuracy and persuasiveness of this method.

\section{Conclusions}

1) In this case, photosynthesis was limited mainly because soybean leaves reduced the photosynthetic efficiency through stomatal regulation or stomatal limitation, in order to reduce or avoided the degree of damage caused by light energy to photosynthetic systems.

2) By analysing chlorophyll fluorescence parameters and podding numbers, Qihuang 35 is the drought-resistant variety and Hedou 12 is the sensitive variety. The actual podding numbers are correlation with chlorophyll fluorescence parameters, especially $Y(I I)$.

3) Compared with the parameters, the chlorophyll fluorescence image can express the photosynthetic information more completely. $Y(I I)$ is the reflection of photosynthetic state of plants and this study provides two algorithms to predict of $Y(I I)$ though chlorophyll fluorescence images, with correlation coefficients are $R=0.85989$ (MLR) and $R=0.84285$ (PLS).

\section{Acknowledgements}

This work was financially supported by the Beijing Academy of Agriculture and Forestry Sciences Program (No. KJCX20170418), Natural Science Foundation of China (31601216) and Beijing Municipal Science and Technology Project (D151100004215002).

\section{[References]}

[1] Wang W F, Peng C H, Kneeshawdaniel D, Larocqueguy R, Luo Z. Drought-induced tree mortality: ecological consequences, causes, and modeling. Environ. Rev., 2012; 20(2): 109-121.

[2] Allen C D, Macalady A K, Chenchouni H, Bachelet D, Mcdowell N, Vennetier $\mathrm{M}$, et al. A global overview of drought and heat-induced tree mortality reveals emerging climate change risks for forests. For. Ecol. Manage., 2010; 259(4): 660-684.

[3] Cheeseman J. 7 - Food security in the face of salinity, drought, climate change, and population growth. Halophytes for Food Security in Dry Lands, 2016; pp.111-123.

[4] Pew Research Center. The future of world religions: population growth projections, 2010-2050. Washington, DC: Pew Research Center, 2015.

[5] Shao G, Huang D, Cheng X, Cui J, Zhang Z. Path analysis of sap flow of tomato under rain shelters in response to drought stress. Int. J. Agric. \& Biol. Eng., 2016; 9(2): 54-62.

[6] Masuda T, Goldsmith P D. World soybean production: area harvested, yield, and long-term projections. Int. Food \& Agribusiness Manage. Rev., 2009; 12(4): 233-236.

[7] Panagopoulos Y, Gassman P W, Arritt R W, Herzmann D E, Campbell T D, Valcu A, et al. Impacts of climate change on hydrology, water quality and crop productivity in the Ohio-Tennessee River Basin. Int. J. Agric. \& Biol. Eng., 2015; 8(3): 1-18.

[8] Golldack D, Li C, Mohan H, Probst N. Tolerance to drought and salt stress in plants: Unraveling the signaling networks. Front. in Plant Sci., 2014; 5(2): 151.

[9] Bollig C, Feller U. Impacts of drought stress on water relations and carbon assimilation in grassland species at different altitudes. Agric. Ecosyst. Environ., 2014; 188(188): 212-220

[10] Zivcak M, Kalaji H M, Shao H B, Olsovska K, Brestic M. Photosynthetic proton and electron transport in wheat leaves under prolonged moderate drought stress. J. Photochem. Photobiol., 2014; 137(8): 107-115.

[11] Singh S K, Raja R K. Regulation of photosynthesis, fluorescence, stomatal conductance and water-use efficiency of cowpea (Vigna unguiculata L. Walp.) under drought. J. Photochem. Photobiol. B, 2011; 105(1): 40-50.

[12] Martinez-Ferri E, Zumaquero A, Ariza M T, Barceló A, Pliego M C. Non-destructive detection of white root rot disease in avocado rootstocks by leaf chlorophyll fluorescence. Plant Dis., 2015; 100(1): 49-58.

[13] Mathur S. Chlorophyll a fluorescence study revealing effects of high salt stress on Photosystem II in wheat leaves. Plant Physiol. Biochem., 2010; 48(1): 16-20.

[14] Baker N R. Chlorophyll fluorescence: a probe of photosynthesis in vivo. Plant Biol., 2008; 59(59): 89-113.

[15] Qian W, Hong S, Li M Z, Wei Y. Development and application of crop monitoring system for detecting chlorophyll content of tomato seedlings. Int. J. Agric. \& Biol. Eng., 2014; 7(2): 138-145.

[16] Ghotbi-Ravandi A A, Shahbazi M, Shariati M, Mulo P. Effects of mild and severe drought stress on photosynthetic efficiency in tolerant and susceptible barley (hordeum vulgare L.) genotypes. J. Agron. \& Crop Sci., 2015; 200(6): 403-415.

[17] Farquhar G D, Sharkey T D. Stomatal conductance and photosynthesis. Annu. Rev. Plant Physiol., 1982; 33(1): 74-79. 
[18] Lu Q Q, Song X S, Yan D H. Effects of drought stress on photosynthetic physiological characteristics in soybean seeding. Chin. Agric. Bull., 2012; 28(9): 42-47. (in Chinese)

[19] Guo S J, Yang K M, Huo J, Zhou Y H, Wang Y P. Influence of drought on leaf photosynthetic capacity and root growth of soybeans at grain filling stage. Chin. J. Appl. Ecol., 2015; 26(5): 1419-1425. (in Chinese)

[20] Lawlor D W, Tezara W. Causes of decreased photosynthetic rate and metabolic capacity in water-deficient leaf cells: a critical evaluation of mechanisms and integration of processes. Ann. Bot., 2009; 103(4): 561-579.

[21] Schreiber U, Schliwa U, Bilger W. Continuous recording of photochemical and non-photochemical chlorophyll fluorescence quenching with a new type of modulation fluorometer. Photosynth. Res., 1986; 10(1-2): 51-62

[22] Maxwell K, Johnson G N. Chlorophyll fluorescence - A practical guide. J. Exp. Bot., 2000; 51(345): 659-668

[23] Shahenshah, Isoda A. Effects of water stress on leaf temperature and chlorophyll fluorescence parameters in cotton and peanut. Plant Prod. Sci., 2015; 13(3): 269-278.

[24] Galmés J, Pou A, Alsina M M, Tomàs M, Medrano H, Flexas J. Aquaporin expression in response to different water stress intensities and recovery in Richter-110 (Vitis, sp.): relationship with ecophysiological status. Planta, 2007; 226(3): 671-681.

[25] Zhou S X, Medlyn B E, Prentice I C. Long-term water stress leads to acclimation of drought sensitivity of photosynthetic capacity in xeric but not riparian Eucalyptus species. Ann. Bot., 2016; 117(1): 133-144

[26] Ivanov B, Edwards G. Influence of ascorbate and the Mehler peroxidase reaction on non-photochemical quenching of chlorophyll fluorescence in maize mesophyll chloroplasts. Planta, 2000; 210(5): 765-774.

[27] Salvatori E, Fusaro L, Manes F. Chlorophyll fluorescence for phenotyping drought-stressed trees in a mixed deciduous forest. Ann. Bot. 2016; 6: 39-49.

[28] Hou W, Sun A H, Chen H L, Yang F S, Pan J L, Guan M Y. Effects of chilling and high temperatures on photosynthesis and chlorophyll fluorescence in leaves of watermelon seedlings. Bio. Plantarum, 2016; 60(1): 148-154.

[29] Atherton J, Nichol C J, Porcar-Castell A. Using spectral chlorophyll fluorescence and the photochemical reflectance index to predict physiological dynamics. Remote Sens. Environ., 2016; 176: 17-30.

[30] Yang J, Gong W, Shi S, Du L, Sun J, Song S, et al. The effect of chlorophyll concentration of paddy rice on the fluorescence spectrum. Spectroscopy \& Spectral Analysis, 2016; 36(10): 3410-3413. 